\title{
Differentiation between chalazion and sebaceous carcinoma by noninvasive meibography
}

\author{
This article was published in the following Dove Press journal: \\ Clinical Ophthalmology \\ 18 September 2014 \\ Number of times this article has been viewed
}

\author{
Yuji Nemoto' \\ Reiko Arita ${ }^{2,3}$ \\ Atsushi Mizota' \\ Yuko Sasajima ${ }^{4}$ \\ 'Department of Ophthalmology, \\ Teikyo University School of Medicine, \\ Tokyo, ${ }^{2}$ Itoh Clinic, Saitama, \\ ${ }^{3}$ Department of Ophthalmology, \\ The University of Tokyo, Tokyo, \\ ${ }^{4}$ Department of Pathology, Teikyo \\ University School of Medicine, \\ Tokyo, Japan
}

Background: Sebaceous carcinoma is notorious for masquerading clinically as other benign lesions such as chalazion. A tool to better differentiate between these two conditions would thus be desirable.

Purpose: To examine the potential application of noninvasive meibography in the differential diagnosis of chalazion and sebaceous carcinoma of the eyelid as a retrospective cross-sectional study.

Methods: Five individuals with chalazion and three patients with sebaceous carcinoma were observed. Noninvasive meibography was performed to visualize the reflectivity and shape of the lesion in each subject.

Results: Noninvasive meibographic imaging revealed chalazion as a lesion of overall low reflectivity with small regions of higher reflectivity corresponding to lipid granules. On the other hand, the noninvasive meibography revealed sebaceous carcinoma as a poorly marginated lesion of high reflectivity in the eyelid.

Conclusion: Noninvasive meibographic imaging may prove useful for the differential diagnosis of chalazion and sebaceous carcinoma. It may also be informative in definition of the resection area in carcinoma patients.

Keywords: noninvasive meibography, sebaceous carcinoma, chalazion, eyelid

\section{Introduction}

Chalazion and sebaceous carcinoma of the eyelid are diseases that originate predominantly from meibomian glands. Chalazion is a relatively common condition characterized by lipogranulomatous inflammation of meibomian glands in the eyelid. It often arises secondarily to noninfectious obstruction of a meibomian gland duct. ${ }^{1}$ Histopathologically, chalazion manifests as a lipogranulomatous reaction to liberated lipid granules contained within a connective tissue pseudocapsule. It is usually managed by the application of a hot compress and good eyelid hygiene. Symptomatic chalazion can be removed by incision and curettage. The prognosis for individuals with chalazion is excellent.

On the other hand, sebaceous carcinoma is a relatively uncommon malignant neoplasm that most often arises in the meibomian glands of the eyelid. It results from the malignant proliferation of sebaceous cells that manifest a vacuolated cytoplasm due to the presence of lipid. Sebaceous carcinoma mostly affects older adults, although it can also develop in younger individuals at 10-20 years of age..$^{2,3}$ Two of the most common clinical presentations of sebaceous carcinoma of the eyelid are a solitary firm eyelid nodule and eyelid thickening associated with chronic blepharoconjunctivitis. Intraepithelial (pagetoid) spread into the eyelid epidermis and conjunctival epithelium is also apparent in some cases. This aggressive neoplasm can exhibit local recurrence as well
Correspondence: Reiko Arita Itoh Clinic, 626-II, Minaminakano, Minuma-ku, Saitama City, Saitama 337-0042, Japan

Tel $+8 \mid 486865588$

Fax $+8 I 486868485$

Email ritoh@za2.so-net.ne.jp 
as regional or distant metastasis, resulting in higher morbidity and mortality. ${ }^{2}$ It is best managed by wide surgical excision with frozen sectioning. If the suspected lesion is large, a punch biopsy or small map biopsies may be initially performed, with subsequent surgery being planned on the basis of the extent of the tumor determined histopathologically. ${ }^{2}$

Sebaceous carcinoma is notorious for masquerading clinically as other benign lesions such as chalazion. In one study, it was the most commonly missed malignancy (1.1\%) among 1,060 cases diagnosed clinically as chalazion. ${ }^{4}$ Other studies have found the rate of misdiagnosis of sebaceous carcinoma as chalazion to be $25.6 \%{ }^{5}$ and $20 \% .^{2}$ Such misdiagnosis can result in a delayed implementation of the correct treatment. A tool to better differentiate between these two conditions would thus be desirable.

Noninvasive meibography (NIM) was developed to observe the morphology of meibomian glands with the use of an infrared filter and infrared charge-coupled device camera (Figure 1).

Such a meibography system allows the structure of meibomian glands to be readily and rapidly (within $\sim 1$ minute) observed in both the upper and lower eyelids without any discomfort to the patient. The meibomian glands appear white (highly reflective) as a result of the presence of meibomian lipid (Figure 1B and C). This approach is helpful not only for the diagnosis of evaporative dry eye but also for detection of morphological changes of meibomian glands associated with meibomian gland dysfunction, ${ }^{5}$ the wearing of contact lenses, ${ }^{6}$ or allergic conjunctivitis. ${ }^{7}$ It was also recently applied in the diagnosis of Peters' anomaly. ${ }^{8}$ Moreover, NIM has previously been applied to the diagnosis of a case of sebaceous carcinoma of the eyelid, with the region of the tumor showing high reflectivity in comparison with the neighboring normal tissue. ${ }^{9}$

In the present study, the efficacy of NIM as a noninvasive diagnostic tool to differentiate between chalazion and sebaceous carcinoma was evaluated.

\section{Methods}

The study subjects included eight patients (eight eyes) with sebaceous carcinoma or chalazion diagnosed on the basis of slit-lamp examination, NIM, and histopathologic findings at Teikyo University (Tokyo, Japan) between August 2011 and June 2013 and evaluated retrospectively. Written informed consent was obtained from all subjects before examination. The study was approved by the institutional review board of Teikyo University and adhered to the tenets of the Declaration of Helsinki. The characteristics of the subjects are shown in Table 1.

External inspection of the eyelids and tarsal conjunctiva was performed at the beginning of each examination with the use of a slit-lamp biomicroscope (TOPCON Corporation, Tokyo, Japan) equipped with a Sony camera. An NIM system (BG-4M; TOPCON) was used to observe and obtain images of the morphology of meibomian glands in both upper and lower eyelids.

In some cases with chalazion, an NIM image of a curettage specimen was performed. Histological specimens
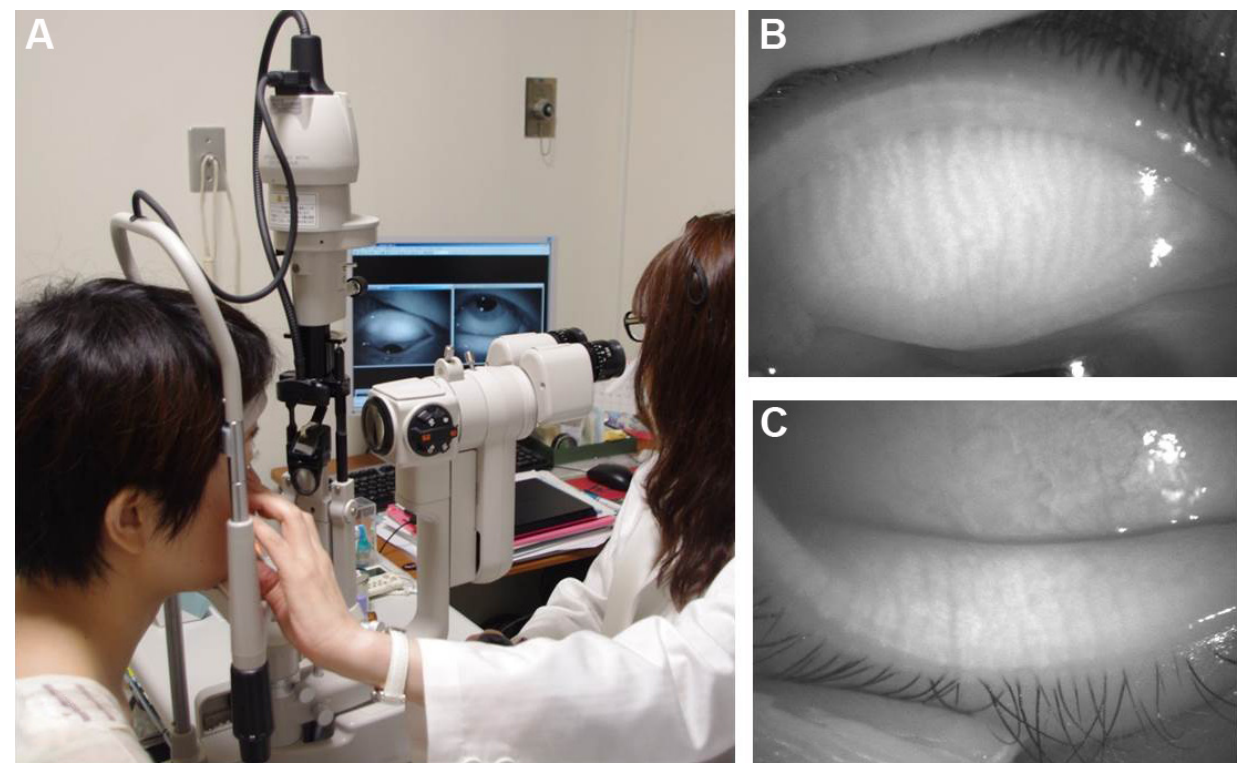

Figure I Noninvasive meibography.

Notes: Photographs demonstrate the $(\mathbf{A})$ meibography system and the $(\mathbf{B})$ right upper eyelid and (C) right lower eyelid in a normal subject. 
Table I Characteristics of the study subjects

\begin{tabular}{|c|c|c|c|c|c|}
\hline Pathological diagnosis & Case number & Age & Sex & Affected eyelid & Duration of condition \\
\hline \multirow[t]{5}{*}{ Chalazion } & 1 & 32 years & Female & Left upper & 6 months \\
\hline & 2 & 29 years & Female & Right lower & 2 weeks \\
\hline & 3 & 34 years & Female & Right upper & 3 months \\
\hline & 4 & 46 years & Male & Right upper & 3 months \\
\hline & 5 & 61 years & Male & Right upper & 5 months \\
\hline \multirow[t]{3}{*}{ Sebaceous carcinoma } & 6 & 87 years & Male & Left upper & 8 months \\
\hline & 7 & 89 years & Female & Left upper & 6 months \\
\hline & 8 & 82 years & Female & Left upper & 2 months* \\
\hline
\end{tabular}

Note: *Recurrence after resection elsewhere.

obtained to verify the diagnosis of sebaceous carcinoma and stained with hematoxylin-eosin were also assessed.

\section{Results}

On examination by NIM, all five cases of chalazion manifested a mostly lower reflectivity of the nodule lesion compared with the surrounding normal tissue, although regions of high reflectivity were also observed within the lesion. No abnormalities outside of the chalazion area were detected by
NIM in any of the five cases. An NIM image of the curettage specimen showed low reflectivity for the granuloma lesion and high reflectivity for fatty granules. Figure 2 (Case 1) shows the low reflectivity of most of the affected area with small regions of high reflectivity. Figure 3 (Case 5) shows a similar pattern but with larger regions of higher reflectivity. Histological analysis revealed a lipogranulomatous lesion containing many foamy macrophages in the curettage granuloma of all chalazion cases.
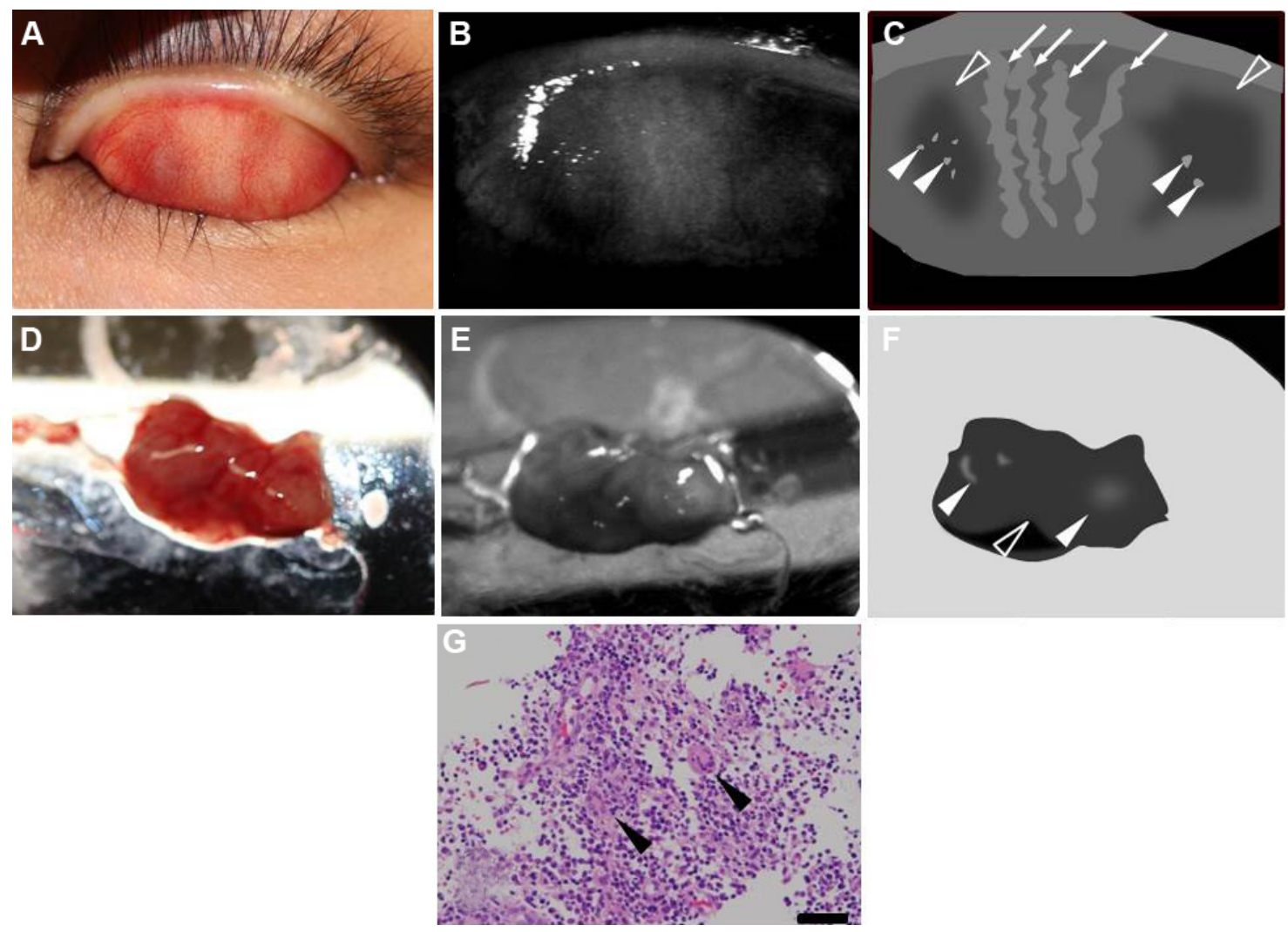

Figure 2 Case I: a 32-year-old woman with chalazion.

Notes: (A) Photograph of the tarsal conjunctiva of the left upper eyelid. Conjunctival injection and swelling at the nasal and lateral aspects were apparent. (B) Noninvasive meibographic image of the tarsal conjunctiva of the left upper eyelid and $(\mathbf{C})$ its schematic representation. Meibomian glands in the central area of the eyelid had a normal appearance (arrows), whereas lesions at the nasal and lateral sides showed a lower reflectivity (open arrowheads) but contained small regions of higher reflectivity (closed arrowheads). (D) Photograph of the curettage specimen at the nasal side. (E) Noninvasive meibographic image of the curettage specimen and (F) its schematic representation. Low reflectivity was apparent for the granuloma lesion (open arrowhead) and high reflectivity for fatty granules (closed arrowheads). (G) Histopathologic analysis of the granuloma lesion. Fatty vacuoles surrounded by epithelioid cells, giant cells (arrowheads), and inflammatory cells were observed (hematoxylin-eosin; bar $=100 \mu \mathrm{m}$ ). 

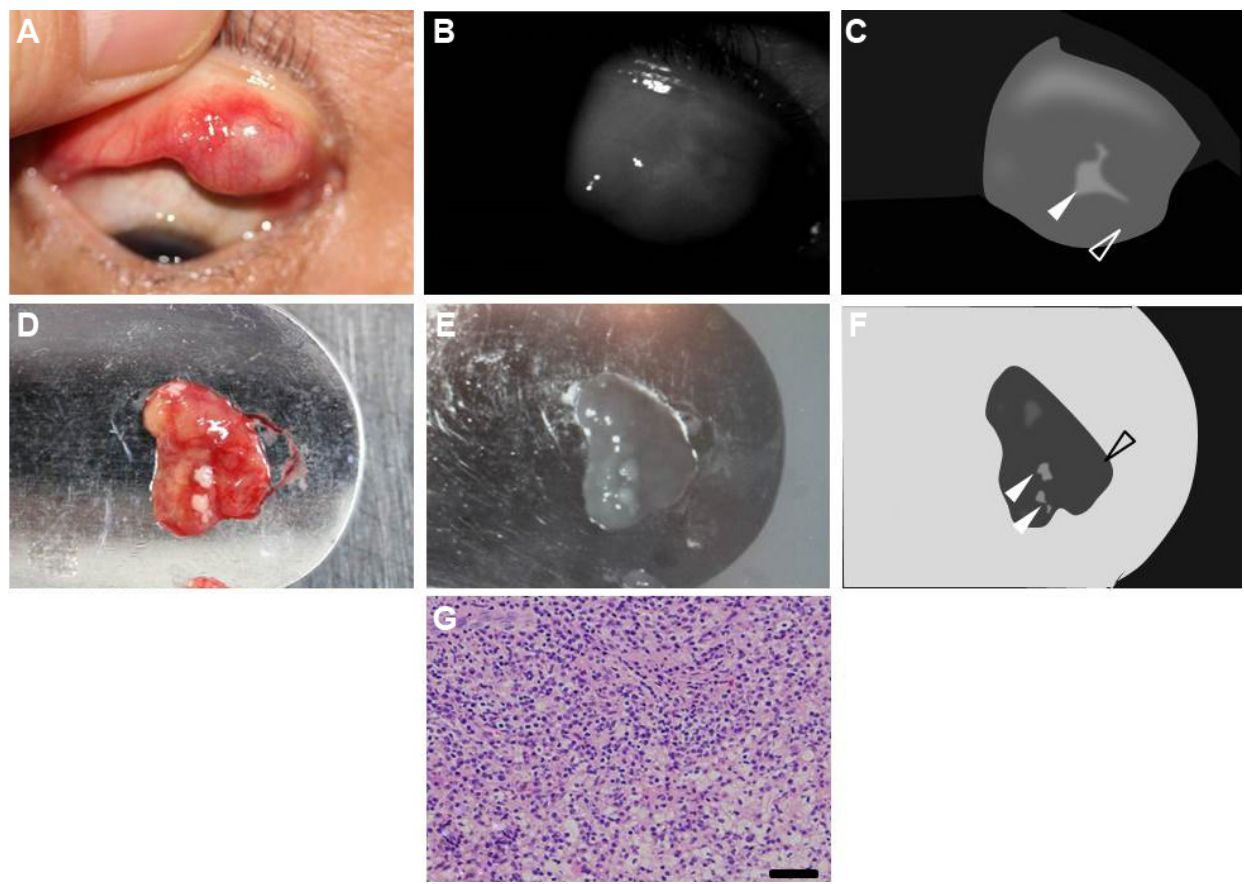

Figure 3 Case 5: a 61-year-old man with chalazion.

Notes: (A) Photograph of the tarsal conjunctiva of the right upper eyelid. A subconjunctival mass was observed at the nasal side. (B) Noninvasive meibographic image of the tarsal conjunctiva of the right upper eyelid and (C) its schematic representation. The mass showed overall low reflectivity (open arrowhead) but contained a central region of high reflectivity (closed arrowhead). (D) Photograph of the curettage specimen including fatty granules. (E) Noninvasive meibographic image of the curettage specimen and (F) its schematic representation. Noninvasive meibography detected overall low reflectivity of the granuloma lesion (open arrowhead) but high reflectivity of fatty granules (closed arrowheads). (G) Histopathologic analysis of the granuloma lesion. Inflammatory cells and dense fatty vacuoles were observed (hematoxylin-eosin; bar =I00 $\mu \mathrm{m}$ ).

All three cases of sebaceous carcinoma exhibited a high reflectivity for the tumor mass surrounded by a region of lower reflectivity in NIM images. The level of tumor reflectivity varied somewhat among cases, however. Slitlamp observation of an 87-year-old man with early-stage sebaceous carcinoma (Case 6) revealed a poorly marginated tumor mass that was coincident with an area of high reflectivity and surrounded by an area of lower reflectivity in NIM images (Figure 4). Both the tumor and neighboring area were resected, with the frozen section procedure revealing that the tumor lesion was removed without any remaining malignant cells at the margins. Many of the tumor cells contained fat vacuoles and were arranged as small nests or in a trabecular pattern. Their nuclei were enlarged or pleomorphic, and they had prominent nucleoli. Mitotic figures were also observed in some of the tumor cells.

An 89-year-old woman with advanced-stage sebaceous carcinoma (Case 7) had a mass lesion with a diameter of $11 \mathrm{~mm}$ at the temporal side of the left upper eyelid (Figure 5). NIM revealed that the poorly marginated mass showed a high reflectivity and had spread to the nasal palpebral conjunctiva. The tumor was surgically removed on the basis of slit-lamp microscopic observation. However, histopathologic examination of the primary resected specimen revealed tumor cells that had invaded into the lid margin and nasal conjunctiva. Additional resection including the lesion revealed as the poorly marginated region of high reflectivity by NIM was therefore required to remove the residual tumor. Histology showed that the tumor was located at the lid margin as an ill-defined nodular lesion. Small islands of tumor cells with fatty vacuoles were detected. The neoplastic cells manifested marked atypia and many mitotic figures.

Finally, an 82-year-old woman with recurrent sebaceous carcinoma (Case 8) manifested an irregularity of the lid margin with nodules (Figure 6). NIM revealed a poorly marginated region of high reflectivity coincident with the nodular lesion. The biopsy specimen was found to contain both normal tissue as well as solid nests of tumor cells with fatty vacuoles, pleomorphic nuclei, and many mitotic figures.

\section{Discussion}

In the present study, five cases of chalazion and three cases of sebaceous gland carcinoma of the eyelid were examined by NIM, and the NIM observations were compared with histological findings in order to identify differences between the two conditions. NIM imaging reveals the acinus and ducts 

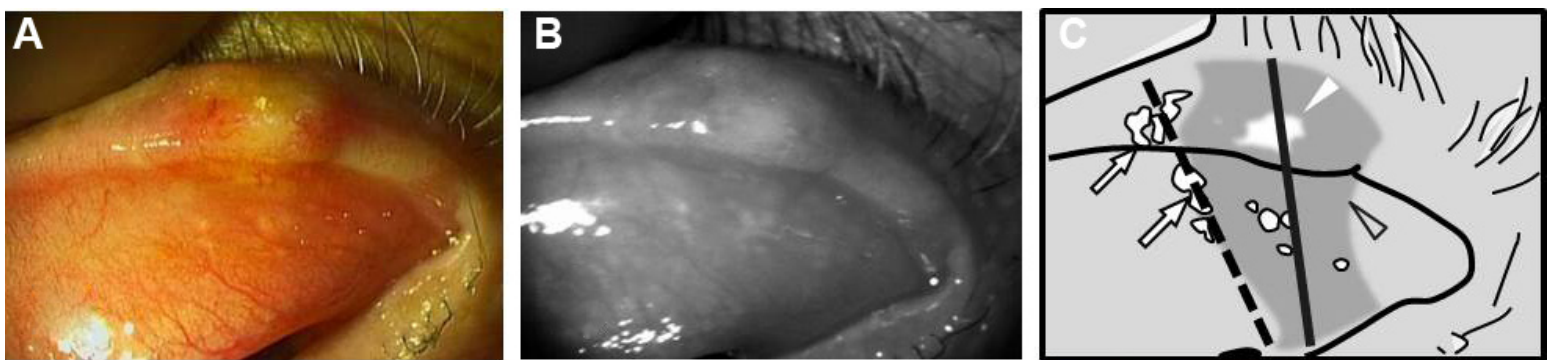

D

E
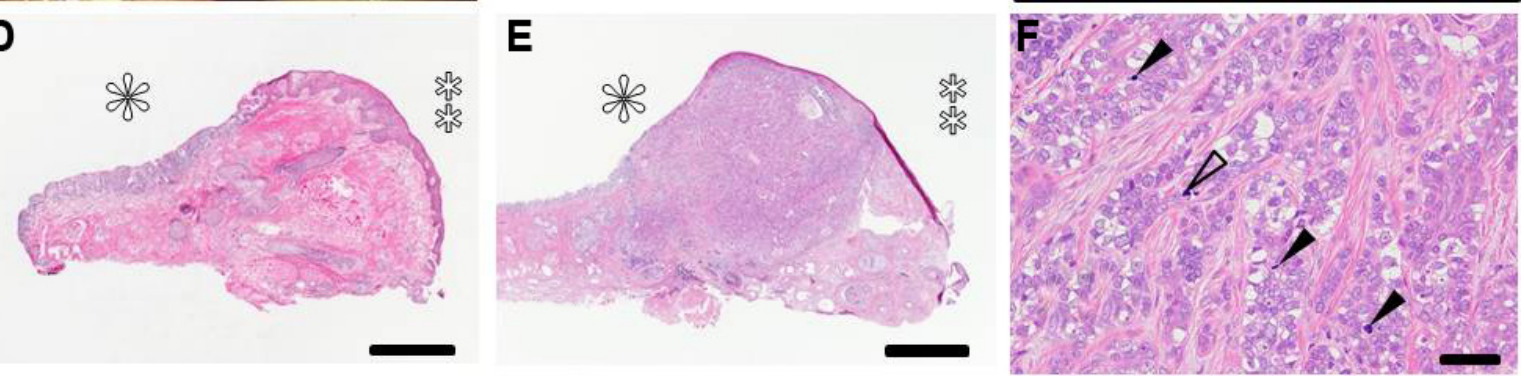

Figure 4 Case 6: an 87-year-old man with sebaceous carcinoma.

Notes: (A) Photograph of the left upper eyelid, revealing a yellow round mass without any defects in eyelashes. The longest dimension of the mass at the eyelid margin was $6 \mathrm{~mm}$. There was no obvious change at the tarsal conjunctiva. (B) Noninvasive meibographic image of the tarsal conjunctiva of the left upper eyelid and (C) its schematic representation. Noninvasive meibography detected a poorly bordered mass lesion with relatively high reflectivity (closed arrowhead), a surrounding region of lower reflectivity (open arrowhead), and sharply marginated granules with high reflectivity (arrows). The dashed line and solid line indicate section lines for pathological observation (see below for further details). (D) Pathological section from the nasal side of the resected specimen. The section line is the dashed line in Figure 4C. The conjunctival (asterisk) and marginal (double asterisk) sides of the eyelid are indicated. No neoplastic cells were evident on the nasal side of the surgical margin, but a normal meibomian gland was apparent (hematoxylin-eosin; bar $=1 \mathrm{~mm}$ ). Pathological section from the central region of the tumor shown at $(\mathbf{E})$ lower and $(\mathbf{F})$ higher magnification. The section line is the solid line in Figure 4C. The conjunctival (asterisk) and marginal (double asterisk) sides of the eyelid are indicated. An ill-defined tumor lesion was observed at the eyelid margin (hematoxylin-eosin; bar $=1 \mathrm{~mm}$ ). The lesion contained small islands of tumor cells with fatty vacuoles. The tumor cells also manifested marked atypia with large and pleomorphic nuclei as well as many mitotic figures (closed arrowheads). Nucleoli were prominent and abnormal mitoses (open arrowhead) were often found (hematoxylin-eosin; bar $=50 \mu \mathrm{m}$ ).
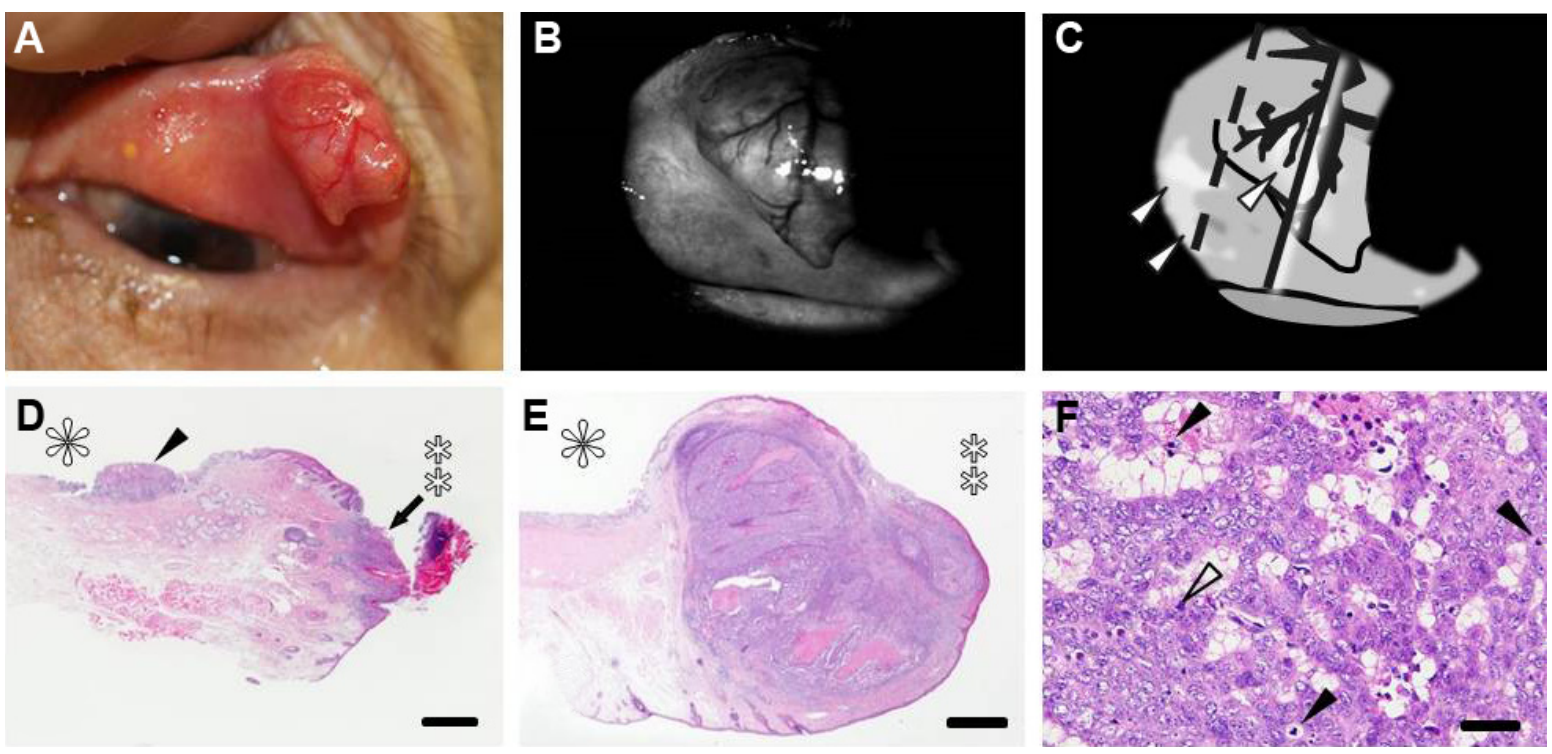

Figure 5 Case 7: an 89-year-old woman with sebaceous carcinoma.

Notes: (A) Photograph of the left upper eyelid. A red round mass with a diameter of II mm was observed. The nasal conjunctiva manifested injection. (B) Noninvasive meibographic image of the tarsal conjunctiva of the left upper eyelid and (C) its schematic representation. Noninvasive meibography detected a poorly bordered mass lesion with relatively high reflectivity (arrowheads) and a surrounding region of lower reflectivity. The dashed line and solid line indicate section lines for pathological observation (see below for further details). (D) Histopathologic section from the nasal side of the primary resected specimen. The section line is the dashed line in Figure 5C. The conjunctival (asterisk) and marginal (double asterisk) sides of the eyelid are indicated. Neoplastic cells were detected in both the eyelid margin (arrow) and conjunctiva on the nasal side (arrowhead) of the surgical margin (hematoxylin-eosin; bar $=1 \mathrm{~mm}$ ). Histopathologic section from the central region of the tumor shown at (E) lower and (F) higher magnification. The section line is the solid line in Figure 5C. The conjunctival (asterisk) and marginal (double asterisk) sides of the eyelid are indicated. An ill-defined tumor lesion was observed at the eyelid margin (hematoxylin-eosin; bar $=1 \mathrm{~mm}$ ). The lesion contained small islands of tumor cells with fatty vacuoles. The tumor cells also manifested marked atypia with large and pleomorphic nuclei as well as many mitotic figures (closed arrowheads). Nucleoli were prominent and abnormal mitoses (open arrowhead) were often found (hematoxylin-eosin; bar $=50 \mu \mathrm{m}$ ). 

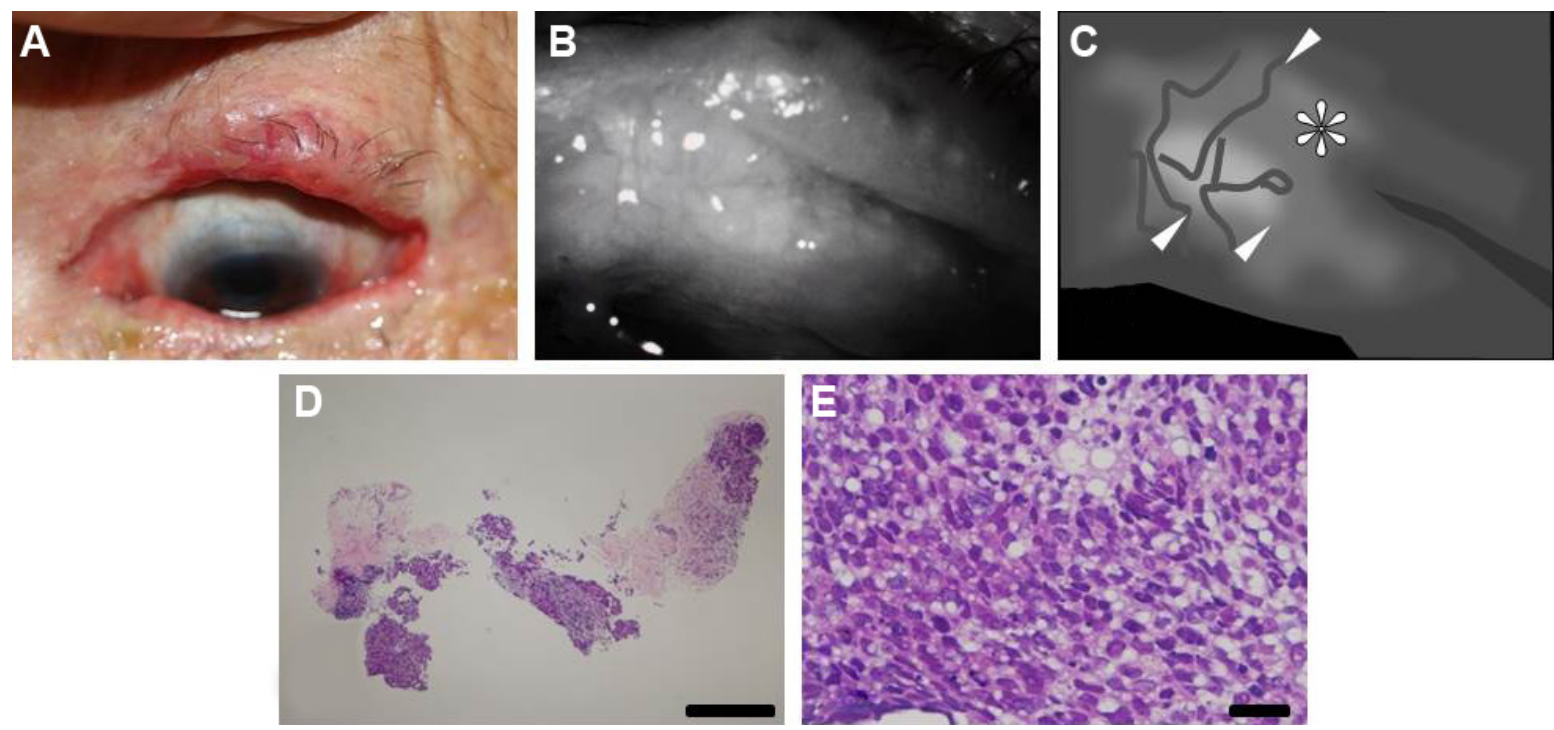

Figure 6 Case 8: an 82-year-old woman with recurrent sebaceous carcinoma.

Notes: (A) Photograph of the left upper eyelid. A nodular lesion with an irregular edge was detected. (B) Noninvasive meibographic image of the tarsal conjunctiva of the left upper eyelid and (C) its schematic representation. Noninvasive meibography revealed a poorly bordered lesion with relatively high intensity in both the nodular region and tarsal conjunctiva (arrowheads). The nodular region of the incisional biopsy is indicated (asterisk). Histopathologic analysis of the incisional specimen from the nodular lesion shown at (D) lower and (E) higher magnification. The nodular region was invaded by tumor (hematoxylin-eosin; bar =l mm). (E) Neoplastic cells with fatty vacuoles were sparsely distributed in the specimen (hematoxylin-eosin; bar $=50 \mu \mathrm{m}$ ).

of normal meibomian glands as sharply bordered regions of high reflectivity. ${ }^{5}$ It was shown that NIM detected chalazion and sebaceous carcinoma as lesions of overall low and high reflectivity, respectively, suggesting that tissue reflectivity visualized by NIM may provide a basis for the differential diagnosis of these two conditions. A schematic representation of the relationship between pathological characteristics and NIM findings for these lesions is shown in Figure 7.
In chalazion, granulomatous tissue comprising infiltrated inflammatory cells and accumulated extracellular matrix replaces the normal meibomian gland and is thus responsible for the low reflectivity in NIM images. Fatty granules present in the nodular lesion are visualized as sharply bordered granular structures of high reflectivity. On the other hand, sebaceous carcinoma with its associated lipid is visualized as a poorly marginated irregular lesion of high reflectivity,

\section{Chalazion}

Higher reflectivity within nodule lesion

\section{Sebaceous carcinoma}

Higher reflectivity

within and without nodule lesion

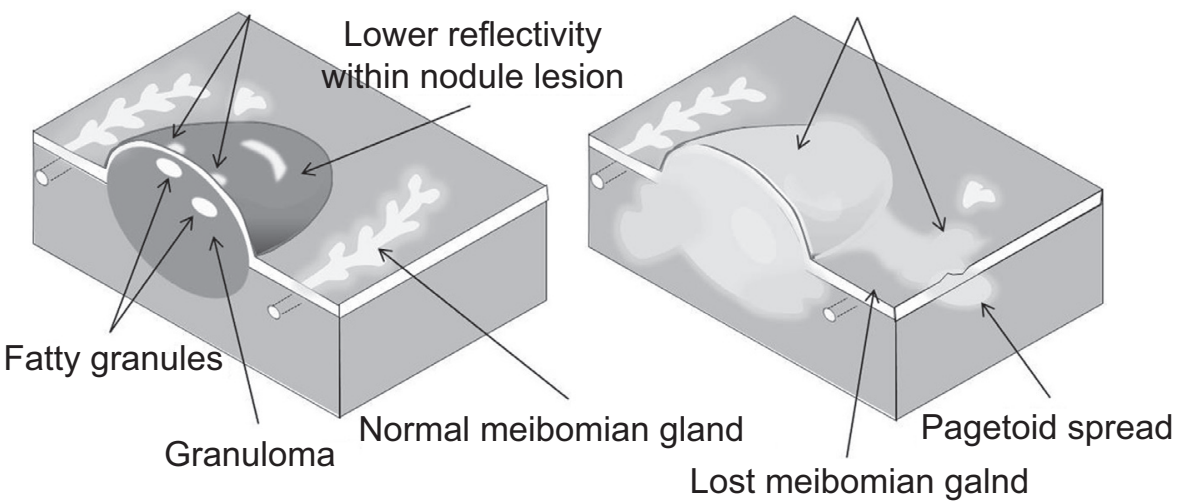

Figure 7 Schematic representation of the relationship between pathological characteristics and noninvasive meibography findings for chalazion and sebaceous carcinoma of the eyelid.

Notes: Chalazion is characterized by lipogranulomatous inflammation and liberated lipid granules within a connective tissue pseudocapsule. The granuloma appears as a lesion of low reflectivity in noninvasive meibographic images, whereas the fatty granules are visualized as sharply bordered structures of high reflectivity within the nodule lesion. Sebaceous carcinoma with its lipid content shows high reflectivity within the nodule lesion by noninvasive meibography. Tumor invasion into the surrounding tissue is also apparent as a poorly marginated irregular-shaped region of high reflectivity. 
which, in some cases, spreads into the surrounding tissue (pagetoid spread). Chalazion and sebaceous carcinoma are thus distinguishable in NIM images on the basis not only of the level of tissue reflectivity but also of the pattern of the reflectivity and the positional relationship between the margin and normal tissue.

Previous reports have emphasized the importance of histopathologic analysis as an invasive examination to prevent misdiagnosis between chalazion and sebaceous carcinoma, ${ }^{2-4}$ while NIM imaging was able to visualize differences between these two conditions in the present study. Given that NIM is a noninvasive and simple tool, it may prove readily applicable to their differential diagnosis. Moreover, NIM imaging may prove informative in planning minimally invasive surgery for sebaceous carcinoma, as exemplified by its detection of the spread of the malignancy to the nasal palpebral conjunctiva in Case 7 (Figure 4).

There are several limitations of this study. First and most important is its small sample size. Second, there was a large age difference between the patients with chalazion and those with sebaceous carcinoma. It will thus be necessary to observe older chalazion patients as well as younger sebaceous carcinoma patients by NIM for a better comparison between the two conditions. Lastly, other tumor types such as basal cell carcinoma and squamous cell carcinoma were not examined. Future studies with larger numbers of patients are thus warranted to address these limitations.

\section{Conclusion}

The results suggest that NIM imaging has the potential to contribute to the differential diagnosis of chalazion and sebaceous carcinoma of the eyelid. It may also prove informative in definition of the resection area in patients with sebaceous carcinoma.

\section{Author contributions}

Design of the study (RA, YN, AM); collection of the data (RA, YN); analysis and interpretation of the data (YN, YS, AM); writing article (YN, RA); critical revision of the article (RA, YS, AM); final approval of the manuscript (AM); provision of materials (RA, YN); obtaining funding (AM); literature search $(\mathrm{YN})$; administrative, technical, or logistic support (RA, AM).

\section{Disclosure}

RA holds a patent for the meibography technique described in this study. The other authors report no conflicts of interest in this work.

\section{References}

1. Shields JA, Shields CL. Eyelid, Conjunctival, and Orbital Tumors. An Atlas and Textbook. 2nd ed. Philadelphia, PA: Lippincott Williams and Wilkins; 2007.

2. Shields JA, Demirci H, Marr BP, Eagle RC Jr, Shields CL. Sebaceous carcinoma of the eyelids: personal experience with 60 cases. Ophthalmology. 2004;111(12):2151-2157.

3. Keskinaslan I, Pedroli GL, Piffaretti JM, Meyer P, Kunz C, Haefliger IO. Eyelid sebaceous gland carcinoma in a young Caucasian man. Klin Monbl Augenheilkd. 2008;225(5):422-423.

4. Ozdal PC, Codere F, Callejo S, Caissie AL, Burnier MN. Accuracy of the clinical diagnosis of chalazion. Eye (Lond). 2004;18(2):135-138.

5. Arita R, Itoh K, Inoue K, Amano S. Noncontact infrared meibography to document age-related changes of the meibomian glands in a normal population. Ophthalmology. 2008;115(5):911-915.

6. Arita R, Itoh K, Inoue K, Kuchiba A, Yamaguchi T, Amano S. Contact lens wear is associated with decrease of meibomian glands. Ophthalmology. 2009;116(3):379-384.

7. Arita R, Itoh $\mathrm{K}$, Maeda $\mathrm{S}$, et al. Meibomian gland duct distortion in patients with perennial allergic conjunctivitis. Cornea. 2010;29(8):858-860.

8. Morishige N, Yamada N, Morita Y, Sonoda KH. Peters' anomaly imaged with an infrared anterior segment camera. Clin Experiment Ophthalmol. 2014;42(4):391-392.

9. Nemoto Y, Mizota A, Arita R, Sasajima Y. [A case with meibomian carcinoma observed in non-invasive meibography]. Folia Japonica de Opthalmologica Clinicam. 2014;7(2):95-99. Japanese.
Clinical Ophthalmology

\section{Publish your work in this journal}

Clinical Ophthalmology is an international, peer-reviewed journal covering all subspecialties within ophthalmology. Key topics include: Optometry; Visual science; Pharmacology and drug therapy in eye diseases; Basic Sciences; Primary and Secondary eye care; Patient Safety and Quality of Care Improvements. This journal is indexed on

\section{Dovepress}

PubMed Central and CAS, and is the official journal of The Society of Clinical Ophthalmology (SCO). The manuscript management system is completely online and includes a very quick and fair peer-review system, which is all easy to use. Visit http://www.dovepress.com/ testimonials.php to read real quotes from published authors. 